\title{
The Pixel Tracking Telescope at the Fermilab Test Beam Facility
}

Simon Kwan ${ }_{a}$, CM Lei ${ }_{a}$, Dario Menasce ${ }_{b}$, Luigi Moroni ${ }_{b}$, Jennifer Ngadiuba ${ }_{b}$, Alan Prosser ${ }_{a}$, Ryan Rivera $a$, Stefano Terzo $_{b}$, Marcos Turqueti ${ }_{a}{ }_{a}$, Lorenzo Uplegger $_{a}$, Luigi Vigani

a Fermi National Accelerator Laboratory, Batavia, IL, USA

b Istituto Nazionale di Fisica Nucleare, Sezione di Milano Bicocca, and Università degli Studi di Milano Bicocca, Piazza della Scienza 3, 20126 Milano, Italy

E-mail: uplegger@fnal.gov

\section{Abstract}

An all silicon pixel telescope has been assembled and used at the Fermilab Test Beam Facility (FTBF) since 2009 to provide precise tracking information for different test beam experiments with a wide range of Detectors Under Test (DUTs) requiring high resolution measurement of the track impact point. The telescope is based on CMS pixel modules left over from the CMS forward pixel production. Eight planes are arranged to achieve a resolution of less than $8 \mu \mathrm{m}$ on the $120 \mathrm{GeV}$ proton beam transverse coordinate at the DUT position. In order to achieve such resolution with $100 \times 150 \mu \mathrm{m}^{2}$ pixel cells, the planes were tilted to 25 degrees to maximize charge sharing between pixels. Crucial for obtaining this performance is the alignment software, called Monicelli, specifically designed and optimized for this system. This paper will describe the telescope hardware, the data acquisition system and the alignment software constituting this particle tracking system for test beam users.

\section{Introduction}

The Fermilab Test Beam Facility (FTBF) at the Fermi National Accelerator Laboratory provides beam in a multitude of particle types and a range of energies with which users can test their detectors [1]. The beam is resonantly extracted in a slow spill for each Main Injector cycle delivering a single 4.2 second long spill per minute. The primary beam (bunched at $53 \mathrm{MHz}$ ) consists of high energy protons (120 $\mathrm{GeV}$ ) at variable intensities between 1 and $300 \mathrm{kHz}$. This beam can also be targeted to create secondary particle beams of pions, muons or electrons with energies down to about $1 \mathrm{GeV}$. Users have access to the facility instrumentation to measure the position and energy of the incident beam. Four pre-installed scintillation counters give rough beam position, a lead glass calorimeters measure the beam energy to a precision of $\sim 3 \%$, two time-of-flight detectors can be set up for particle identification, and finally a silicon pixel telescope provides a precision position measurement of less than $8 \mu \mathrm{m}$ using the primary $120 \mathrm{GeV}$ proton beam. The silicon pixel telescope will

1 Now at Lawrence Berkeley National Laboratory, Berkeley, CA, USA 
be described in this paper along with its data acquisition (DAQ) system and alignment software.

This facility adds to other operating test-beam facilities equipped with high precision tracking telescopes as those of the AIDA project in Europe [2] [3]. The main features of the present one are: a high-energy bunched proton beam (limited multiple Coulomb scattering effects at $120 \mathrm{GeV}$ and precise time localization of the incoming protons), a narrow event time-window (within $\sim 38 \mathrm{nsec}$ ), fast readout system capable to accumulate up to 600,000 beam tracks per minute during the 4.2 second spill, and beam tracking with better than $8 \mu \mathrm{m}$ precision at the DUT.

\section{The Pixel Telescope}

A CMS pixel-based telescope has been built to provide precision tracking information to any test beam experiment at the FTBF that requires the particle impact point on their detector under tests (DUTs) with accurate precision. The telescope is placed along the FTBF beam line and consists of eight detector planes - each made of modules left over from the CMS Forward Pixel detector production - mounted on a carbon fiber frame. Four of the eight telescope planes use modules composed of six $(2 \times 3)$ PSI46V2 Read Out Chips (ROCs) [4], while the remaining four planes are equipped with $2 \times 4$ modules. One of the $2 \times 4$ modules is shown in Figure 1, left side,
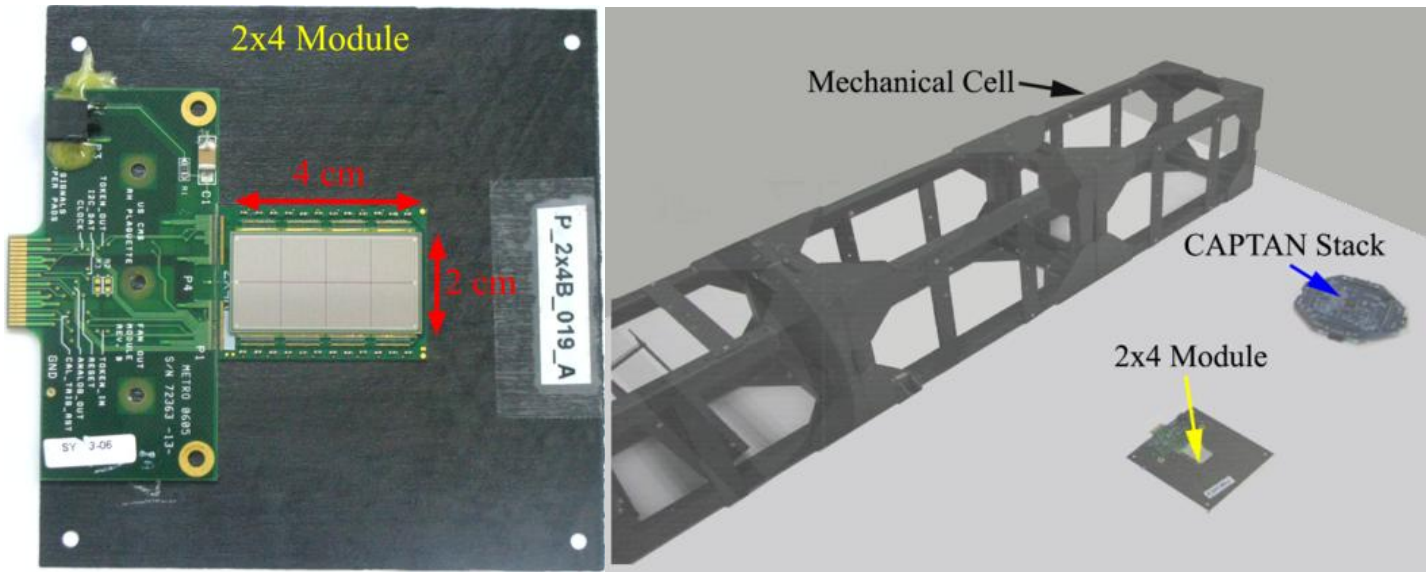

Figure 1. Left: CMS PSI46V2 module. Right: telescope mechanical structure together with its components.

Each ROC reads an array of $52 \times 80$ pixel cells, where each pixel cell size is $100 \times$ $150 \times 285 \mu^{3}$ (except for the pixels at the edge columns and upper row which have sizes $100 \times 300 \times 285 \mu^{3}$ and $200 \times 150 \times 285 \mu^{3}$, respectively), for a total square active area of $0.81 \times 0.81 \mathrm{~cm}^{2}$. The total active area for a $2 \times 4$ module is then $1.62 \times$ $3.24 \mathrm{~cm}^{2}$ while the $2 \times 3$ module total active area is $1.62 \times 2.43 \mathrm{~cm}^{2}$. The eight planes are arranged in two stations with a section available in between for the DUT. The DUT is considered as a separate third station itself.

A three-dimensional schematic view of the telescope detector planes is presented in Figure 2, where the laboratory coordinate system is also indicated. In this reference frame the $\mathrm{Z}$ axis is along the beam direction with $+\mathrm{Z}$ pointing downstream. In order to improve the spatial resolution the planes are tilted by 25 degrees to maximize the clusters of two adjacent hits, in particular: 
- Four planes $(2 \times 4$ detectors $)$ tilted around the $X$ axis with the long pixel side oriented in the $\mathrm{X}$ direction;

- Four planes $(2 \times 3$ detectors $)$ tilted around the $\mathrm{Y}$ axis with the long pixel side oriented in the $\mathrm{Y}$ direction.

In the first case, the most precise measurement is in the $\mathrm{Y}$ coordinate, while in the second case the most precise measurement is in the $\mathrm{X}$ coordinate. This geometry gives a total overlap active area of $\sim 1.6 \times 1.6 \mathrm{~cm}^{2}$.

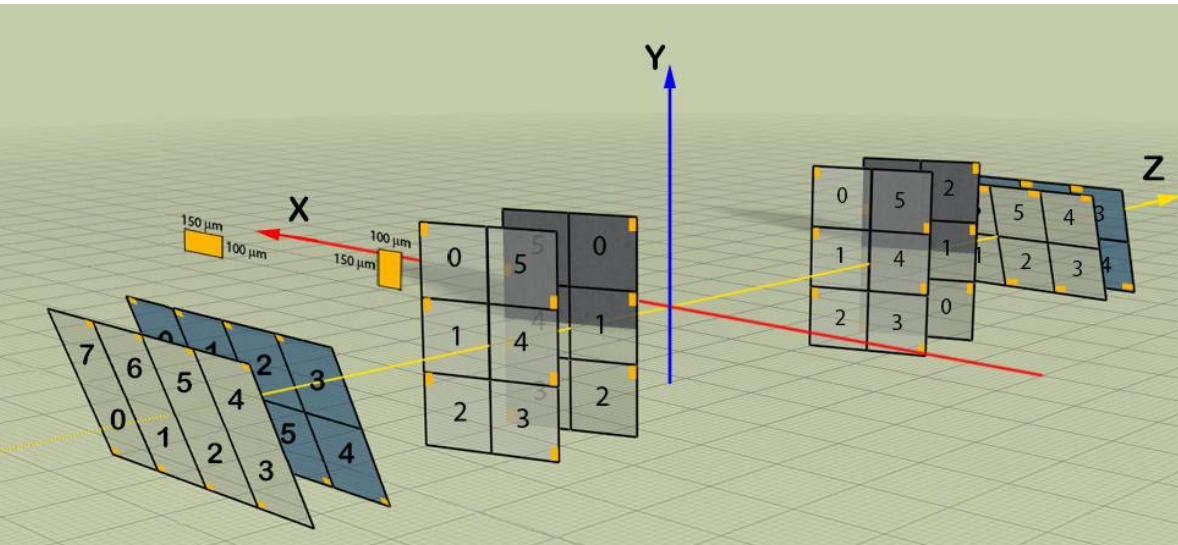

Figure 2. Three-dimensional schematic view of the pixel telescope geometry.

Each detector has a carbon fiber layer with the module glued on it. Heat is passively dissipated through the carbon fiber and hence no active cooling is required. For each station, the detectors are grouped into two pairs and each pair is attached on a light aluminum mechanical support mounted on the mechanical structure of the telescope, as shown in Figure 3.

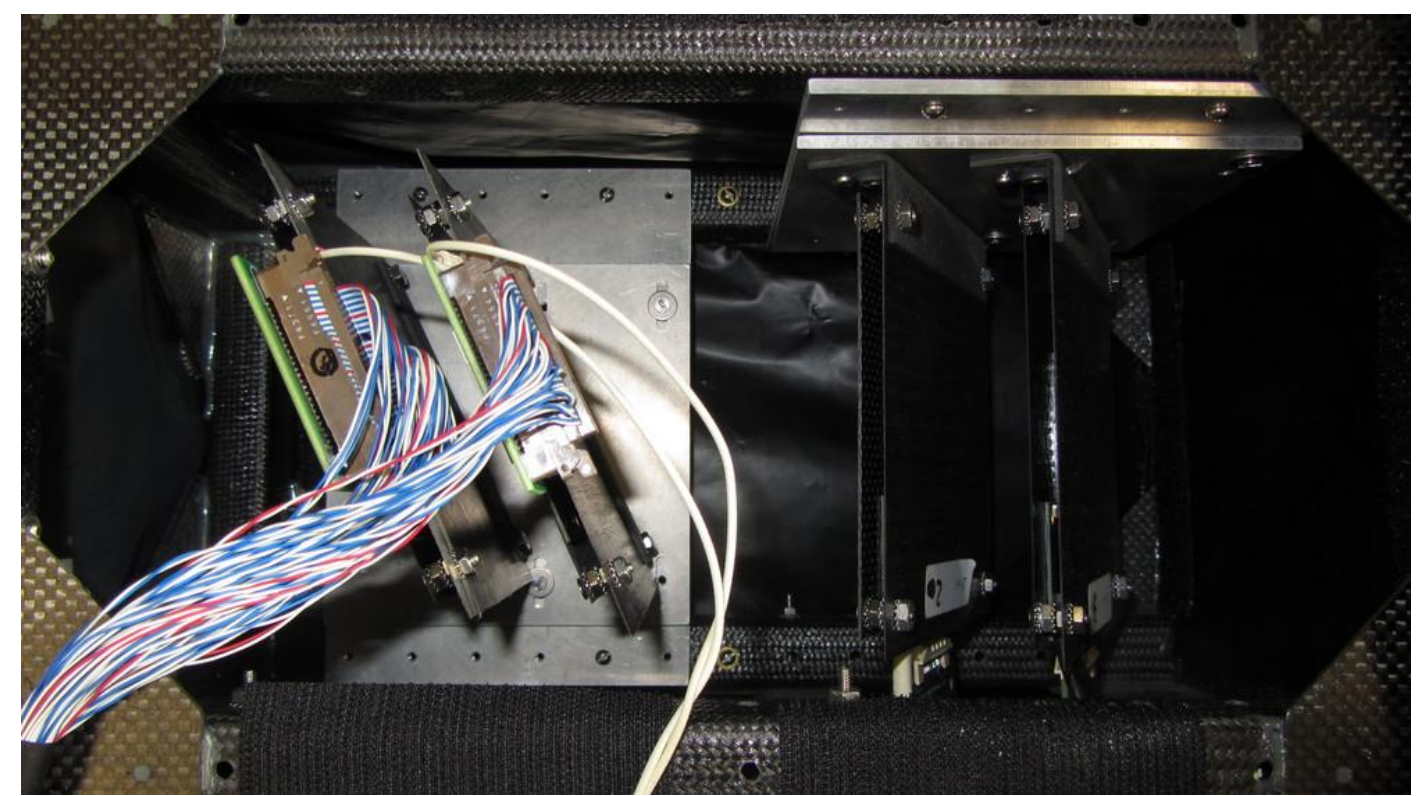

Figure 3. Modules mounted on the aluminum support at 25 degrees inside the carbon fiber frame.

The mechanical structure has a modular design: the frame consists of three basic cells, 
one for each station, built with carbon fiber tubes of dimension $17.0 \times 17.0 \times 34.0$ $\mathrm{cm}^{3}$. The frame is covered by a Mylar anti-static layer, as can be seen in Figure 4, which also serves the secondary purpose of keeping the detectors dark.

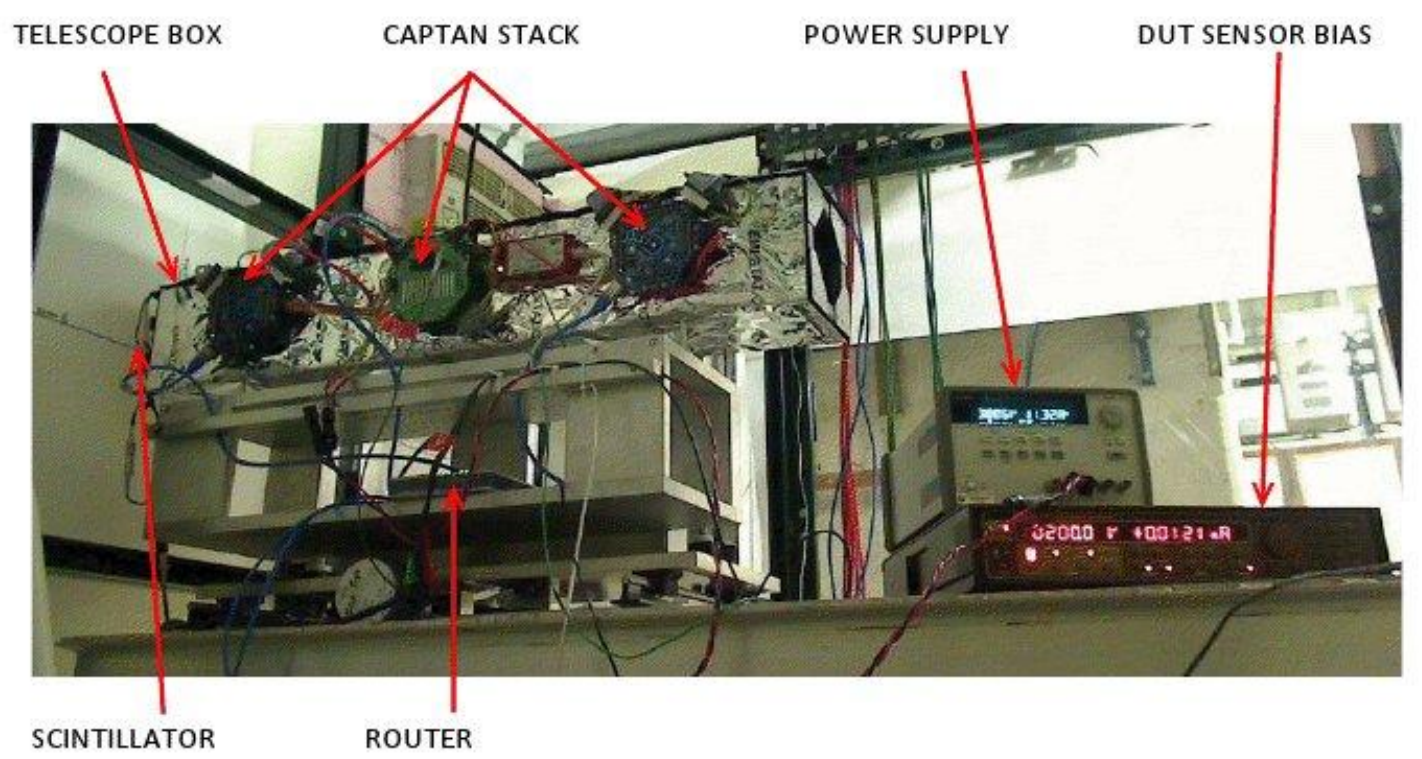

Figure 4. The CAPTAN pixel telescope complete assembly in situ at the FTBF.

The beam particle is triggered by a coincidence signal generated from three scintillation counters placed behind the telescope. The trigger signal opens a small time window in which the data acquisition system collects and sends data from the detectors to the computer. The data from each ROC are tagged with the trigger count, so that time correlated data recorded from all the detectors have the same tag. The hits that share the same trigger count are identified as an event. An event may contain hit data associated with one or more particle tracks passing through the telescope.

To keep the ROCs synchronized with the particle beam, the accelerator clock signal is fed into one of the stations and then redistributed to the other stations through SATA cables. Since the CMS ROC has been designed to run at a maximum frequency of 40 $\mathrm{MHz}$ while the Main Injector accelerator frequency is $53 \mathrm{MHz}$, the clock that is distributed to the stations runs at $26.5 \mathrm{MHz}$, half of the Main Injector frequency, allowing the detector to work properly and remain synchronized with the beam.

\section{The Data Acquisition System}

\subsection{The DAQ: hardware}

The Data Acquisition (DAQ) hardware is based on the CAPTAN system developed at Fermilab [5]. The CAPTAN (Compact And Programmable daTa Acquisition Node) is a flexible and versatile data acquisition system designed to meet the readout and control demands of a variety of pixel and strip detectors for high energy physics applications.

The system consists of three CAPTAN nodes, one for each station. The node is a stack of different function boards with a vertical bus for high-speed data exchange and features a Gigabit Ethernet Link (GEL) for high-speed communication through the network.

In the present case, two boards are stacked in each CAPTAN node (Figure 5): 
- The Node Processing and Control Board (NPCB) provided with a Virtex-4 FPGA.

- The Data Conversion Board (DCB) provided with a 12-bit MAX1438 Analogue to Digital Converter.
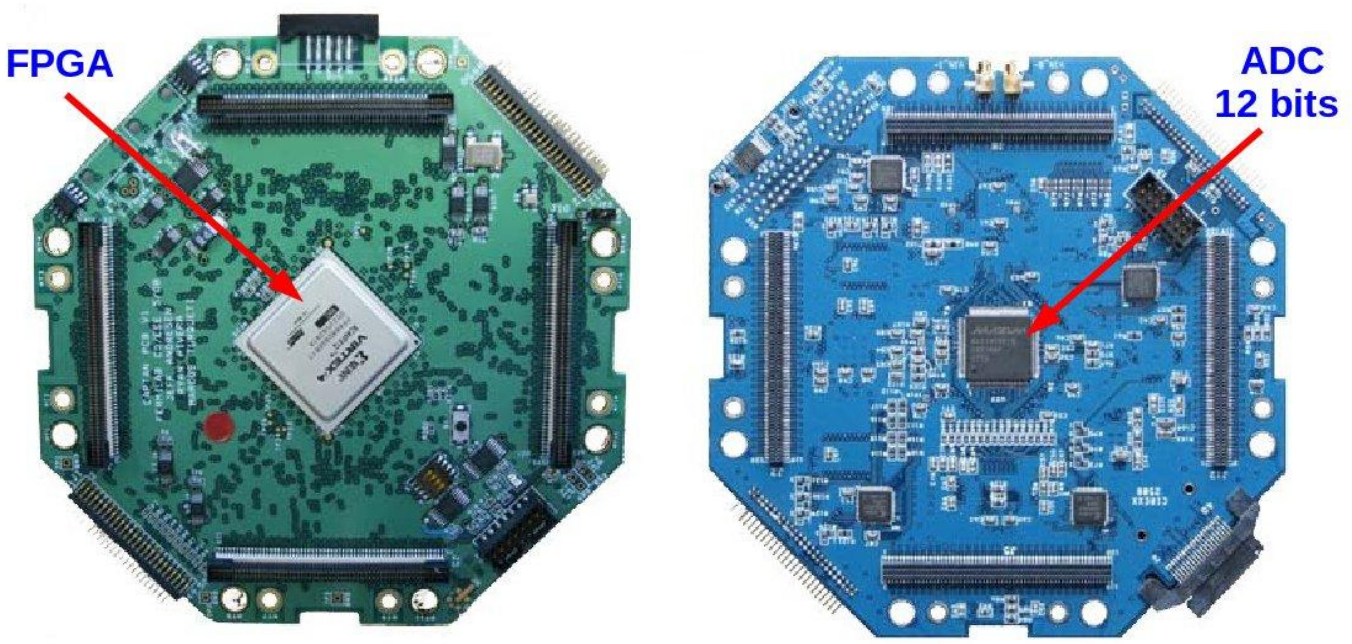

Figure 5. Pictures of the primary boards of the CAPTAN stack used for telescope data acquisition. Left: Node Processing and Control Board. Right: Data Conversion Board.

Each NPCB is connected to a gigabit Ethernet router and the router is, in turn, connected to the computer placed in the FTBF control room through an Ethernet cable. This computer runs the Graphical User Interface, which controls the entire telescope system.

The master CAPTAN node, which connects to the DUTs, receives the accelerator clock and the trigger from the scintillators and redistributes them to the other two nodes after having reduced the clock to half the frequency $(26.5 \mathrm{MHz})$.

In each node, the data from ROCs are received by the DCB, digitalized by the ADC, and then sent to the FPGA through the vertical bus. The formatted data are then transferred to the control room PC via gigabit Ethernet. The diagram in Figure 6 shows the full readout system schematically.

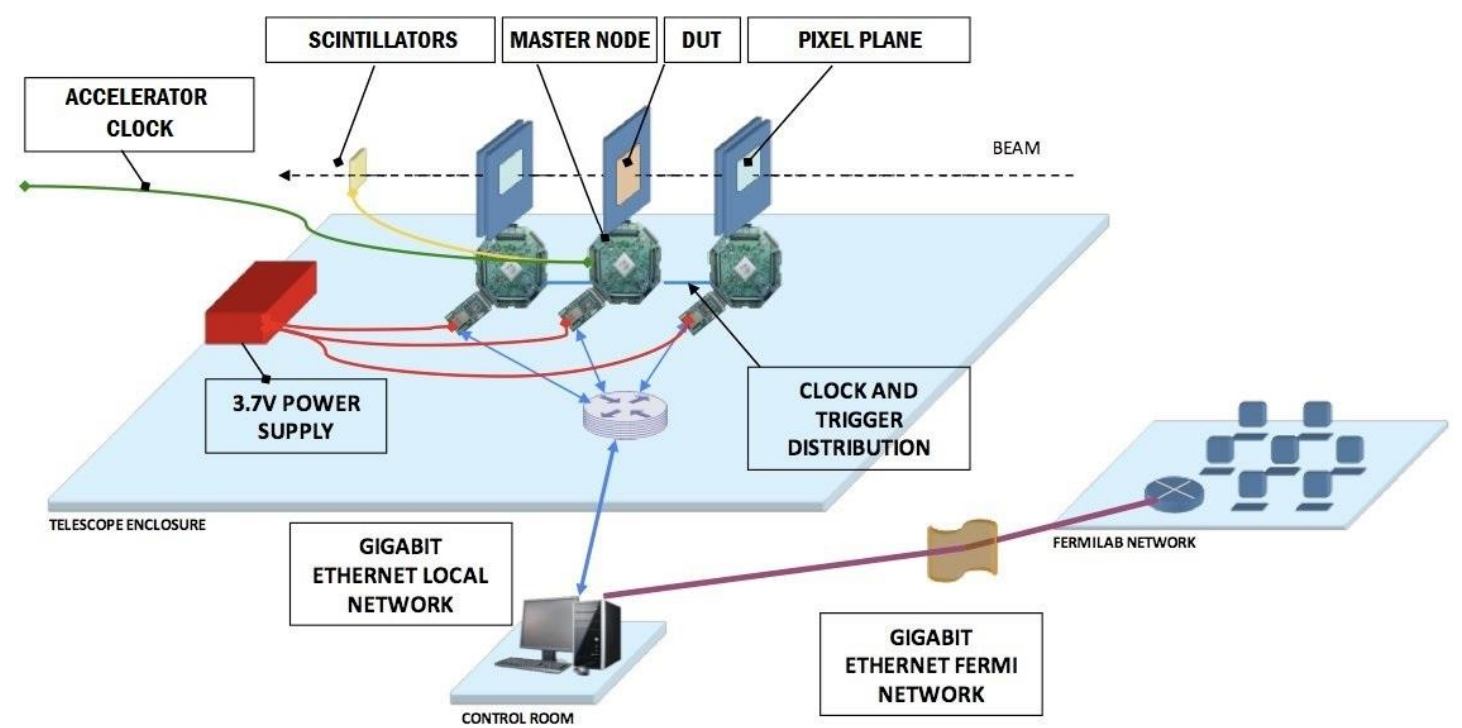

Figure 6. Schematic of the telescope readout system based on CAPTAN hardware. 
At the control room PC, the data from each CAPTAN node are saved in a binary file for each Run. At the end of a Run, the CAPTAN Analysis and Display software, described in the next paragraph, scans the files from the different nodes and merges all temporally correlated data from every pixel plane (marked with the same trigger count) to form a representation of an event. A single binary file containing the merged events is then created for each Run and saved on disk. Each pixel data are stored in a 64 bit word ( 8 bytes), so the typical size of a raw data event for a single telescope track with clusters made of two-pixel hits on all eight planes is only about 128 bytes. A typical Run with 400000 events at a beam-particle rate of $\sim 25 \mathrm{kHz}$ has an average $\sim 0.9$ reconstructed telescope-tracks per event and is of the order of 50 Mbytes including the hits on the DUT. Even at this low beam-particle rate, it requires only $\sim 5$ spills ( $\sim 5$ minutes) to be accumulated. The system has been tested to work up to beam rates of $\sim 150 \mathrm{kHz}$ without any performance degradation, approaching the maximum trigger frequency that the readout chips can tolerate with low hit occupancy.

\subsection{The DAQ: software}

A complete software solution for interfacing with the CAPTAN system has been designed for Microsoft Windows using Microsoft Visual C++ 2008 [6].

The diagram in Figure 7 shows the CAPTAN software topology conceptually. The building blocks of the software are the Global Master (GM), the CAPTAN Controller (CC), a Graphical User Interface (GUI) and the CAPTAN Analysis and Display

179

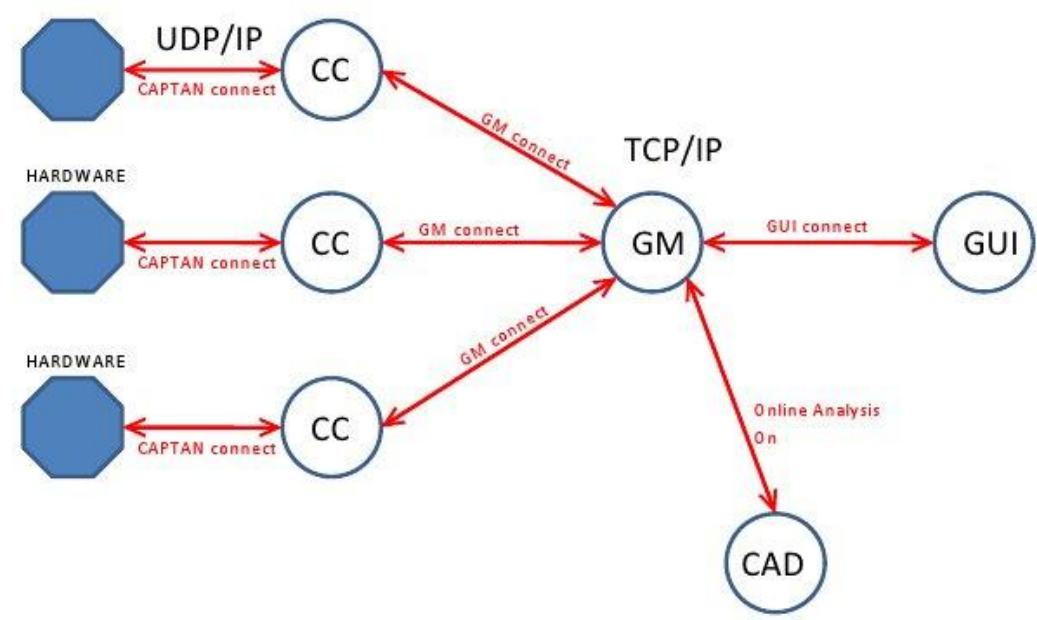

Figure 7. Schematic of the telescope readout system based on CAPTAN hardware.

The GM is the server for the entire system. It handles the communications among the CCs, the GUI and the CAD. The GUI allows the user to set up the readout chips, trigger and clock system, run calibration procedures, as well as to start or stop the data acquisition. It also controls when the $\mathrm{CC}$ stores data to disk. Another crucial block of the software is represented by the CAD, which allows the user to immediately visualize the merged telescope data in three dimensions as shown in Figure 8. This is critical for the data acquisition process since it allows the user to quickly check the quality of the data. For instance, this feature makes it possible to assess whether the beam is properly positioned on the detectors. 


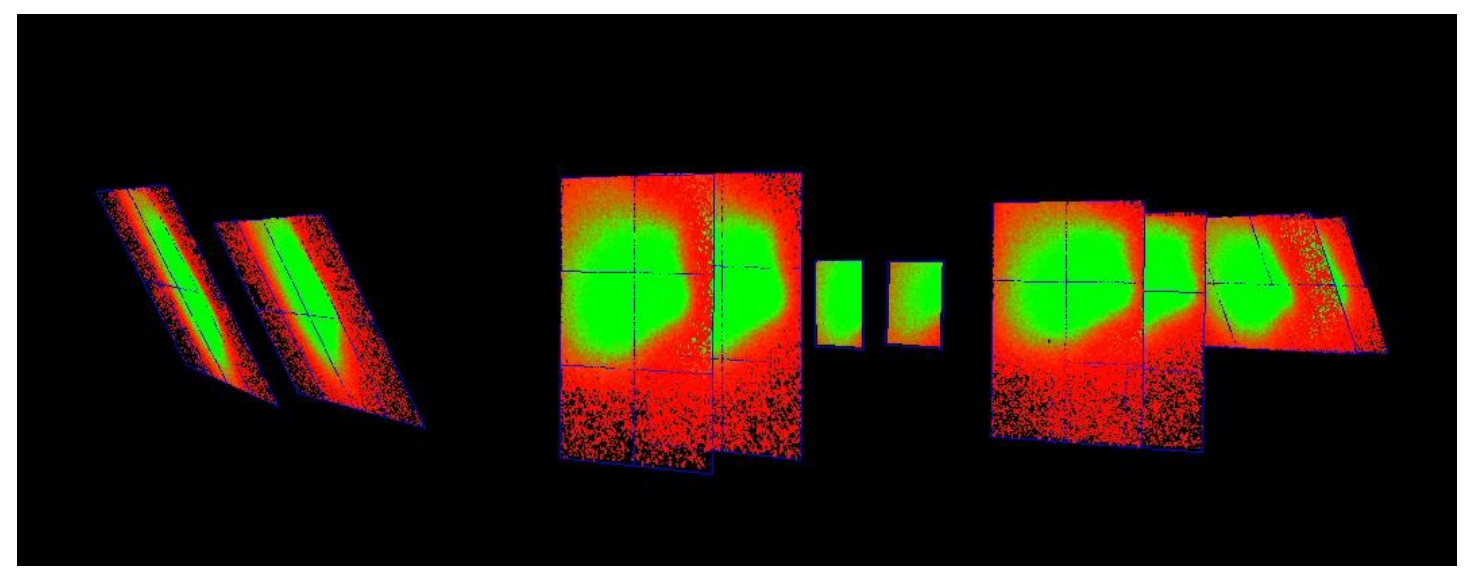

Figure 8. Three-dimensional data visualization with the CAPTAN software.

\section{Telescope Tracking and Alignment}

The main goal of the test beam experiments is to probe the performance of the DUT with a set of well reconstructed beam tracks. For this purpose the telescope has been designed to achieve an optimal resolution on the coordinates of the track impact point on the DUT, which is placed at the center of the telescope. The track reconstruction and the telescope alignment are performed by means of the $\mathrm{C}++$ Monicelli application, developed by the INFN Milano-Bicocca group. This software provides the user with an appropriate iterative procedure to converge toward the optimal alignment of the telescope. All the operations can be accomplished in steps through a graphical interface (GUI) that makes the software user-friendly. For debugging, the GUI allows the user to perform the individual steps of the alignment, change critical parameters interactively and finally monitor the partial results by means of specific histograms and distributions. The software is also equipped with efficient interactive tools allowing the user to browse, examine, print and save these distributions in realtime. Figure 9 shows a snapshot of GUI and its components.

The next paragraphs give a quick overview of the main features of the track reconstruction and alignment procedure. It is worth noting that all the quoted results are for track having hits on all eight planes. 


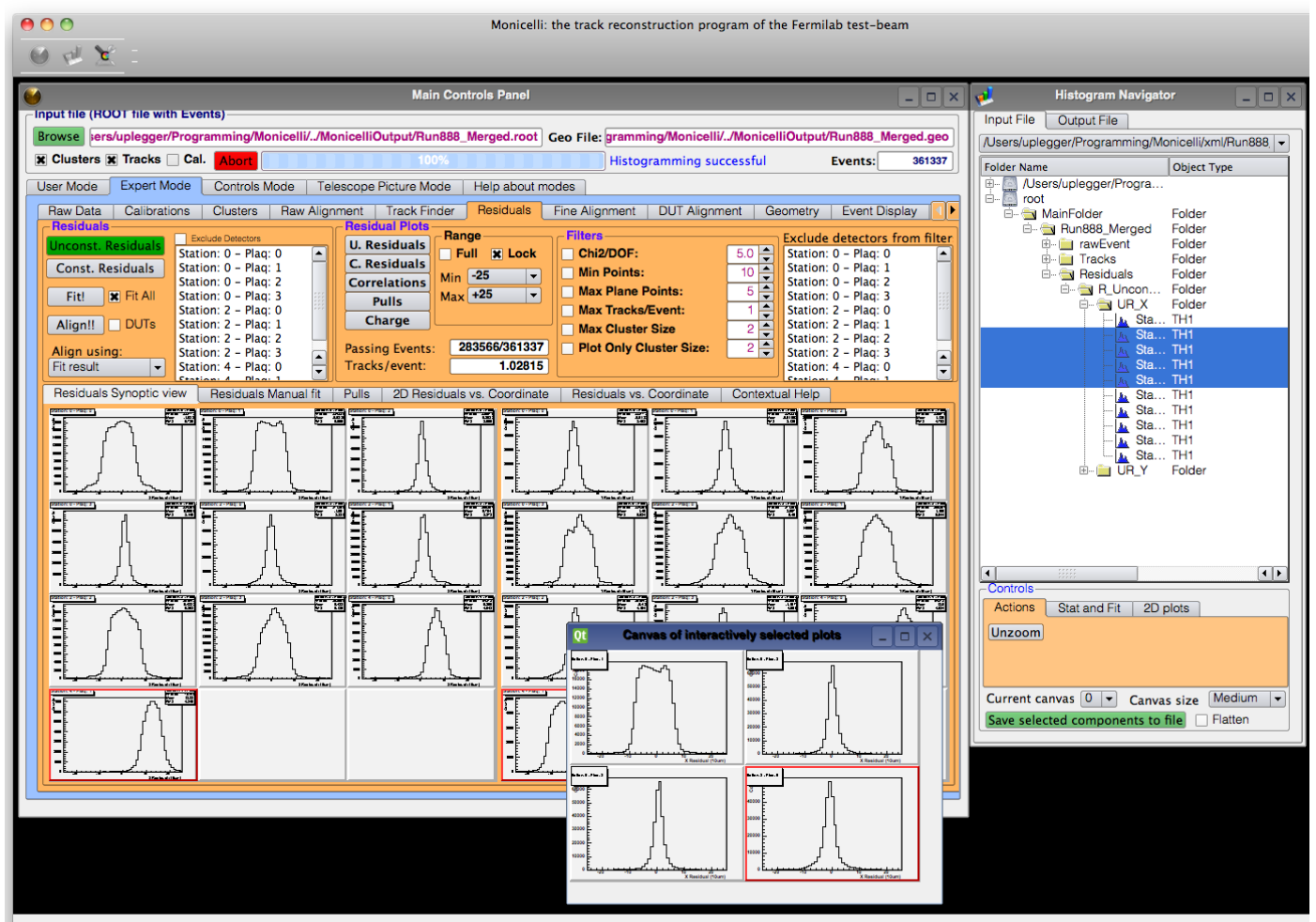

Figure 9. Monicelli Graphical User Interface. Inside the desktop style GUI, the main panel on the top left shows controls for all alignment operations and on the right the histogram browser used to navigate across all control histograms created during the alignment phases. Histograms can be viewed inside the control panel and also in separate canvases, on the bottom, opened by the histogram browser.

\subsection{Track Reconstruction}

The track-reconstruction code implemented in Monicelli was developed for the purpose of this pixel telescope. It performs a simple and fast $\chi^{2}$ straight line fit to the coordinates of the arrays of hits, which were preselected by a pattern recognition based on their alignment. In case of clusters of adjacent hits, the coordinates are linearly interpolated over the expected charge-sharing width. The errors attributed to the coordinates were calibrated during the first commissioning phase of the telescope. They were initially estimated on the basis of what one would a priori expect for the different cluster topologies. Once the alignment was performed they have been refined, by an iterative procedure, which continued until the unconstrained pulls on each telescope plane became Gaussian with nearly unitary RMS. The unconstrained (or unbiased) residuals and pulls of a track on a plane are calculated excluding the hits on that particular plane from the track fit.

To perform this track reconstruction process, Monicelli reads the merged binary data file together with an XML geometry file describing the overall configuration and geometrical details of the telescope detectors for that particular data set. The user can change this file by means of a graphical XML editor included in the package. The geometrical details of each plane are set specifying its space coordinates and rotation angles in the laboratory frame, as well as the number and orientation of ROCs and, for each ROC, the number of columns and rows together with their pitch.

The Monicelli output file consists in a ROOT TTree containing, for each event, the reconstructed telescope tracks together with the associated clusters of hits and the raw data, including those of the DUT. This file provides the user with all the information 
needed for the analysis of the test-beam data. A general-purpose analysis program is also available and can be easily adapted to the particular features of the DUT.

\section{4}

\subsection{Alignment}

The alignment code was expressly developed for this telescope and it is based on a standard $\chi^{2}$ successive-approximation minimization of the unconstrained residuals on each plane. It returns all the six geometrical degrees of freedom of each plane. Only the tracks having hits on all the planes are considered in this process. Typically, no more than 10 iterations with 40000 events are required to converge to a reliable solution.

Figure 10 shows the two unconstrained residuals on a detector plane after a complete alignment. The narrow residual on the left is dominated by charge sharing between adjacent pixels along the tilted coordinate that is measured with the best resolution (pitch $=100 \mu \mathrm{m}$ ), resulting in distributions with RMS of $\sim 16.5 \mu \mathrm{m}$. The other is mainly driven by single-hit events along the non-tilted coordinate (pitch $=150 \mu \mathrm{m}$ ) and, therefore, presents a RMS of about $150 / \sqrt{ } 12 \mu \mathrm{m}$.
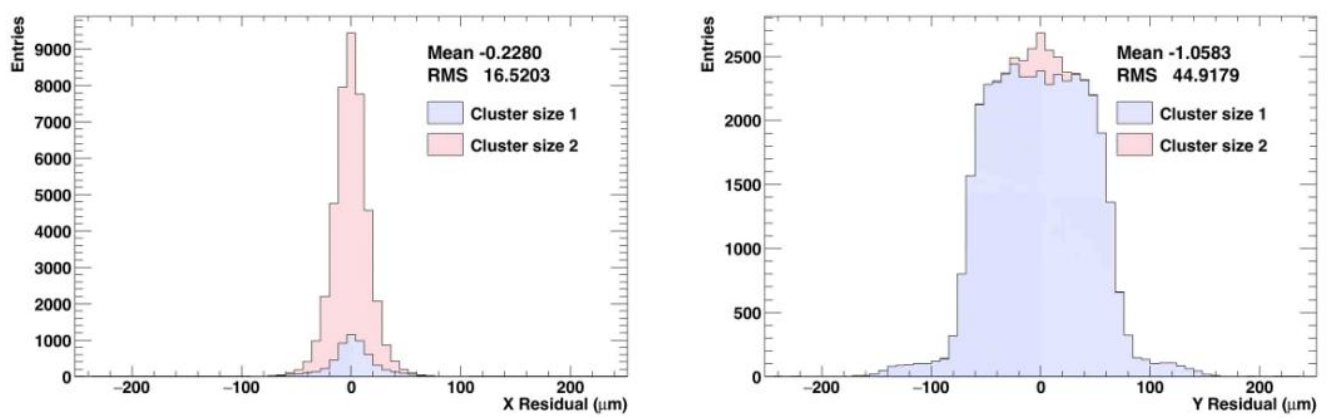

Figure 10. The $x$ and $y$ unconstrained residual distributions for one telescope detector after a complete alignment. $x$ and $y$ are the local coordinates of the plane.

Figure 11 shows the two unconstrained pull distributions on a detector plane after a complete alignment of the telescope. The quasi unitarity of the $x$ and $y$ pull distributions confirms both the precision of the alignment and the correct evaluation of the errors associated to the different clusters of adjacent hits.
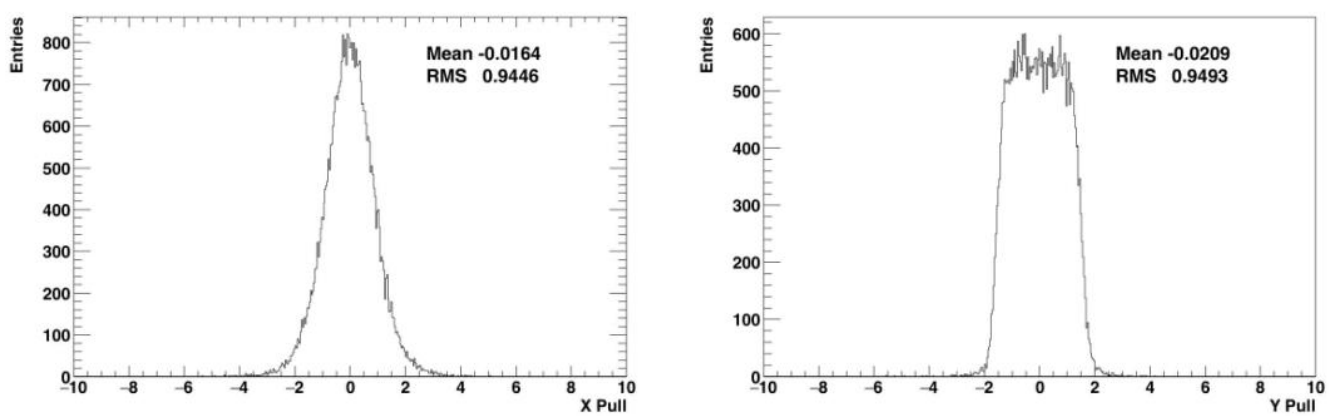

Figure 11. The $x$ and $y$ unconstrained pull distributions for one of the telescope detectors

The accuracy of the alignment is further investigated studying the plots of Figure 12, showing the correlations between the unconstrained residuals and the impact point coordinates on the detector. Any deviation from a flat distribution would signal a residual problem in the alignment. As the alignment procedure goes ahead, the correlation plots are flattened and resemble those shown in Figure 12. 

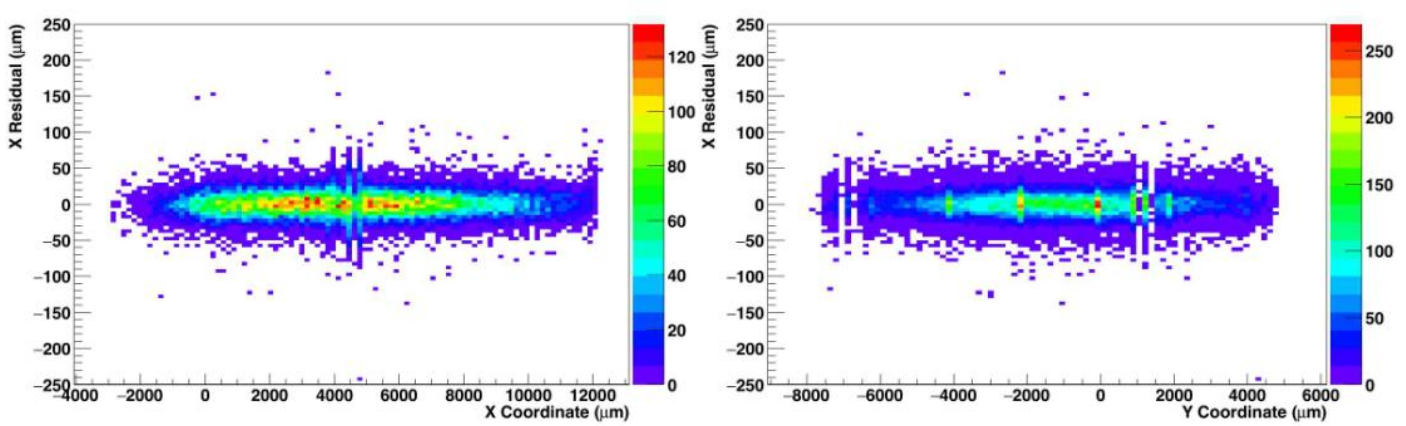

Figure 12. Plots of the correlation between unconstrained residuals and impact point coordinate for one of the telescope detectors.

The shape of the fitted track $\chi^{2} / \mathrm{DoF}$ distribution is shown in Figure 13.

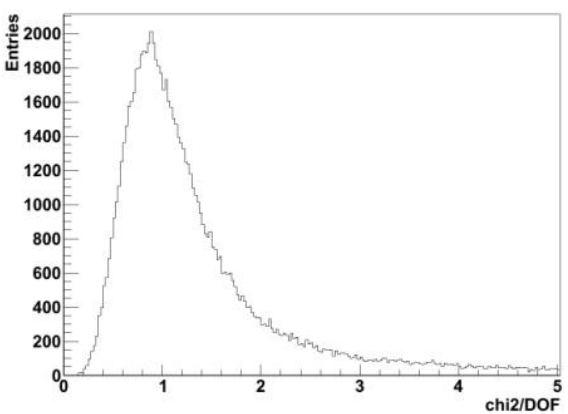

Figure 13. The track $\chi^{2} / \mathrm{DoF}$ distribution after a complete alignment.

Finally, the transverse error on track fit extrapolation at DUT $Z$ position $(Z \approx 0)$ is plotted in Figure 14 (for tracks having hits on all eight planes).
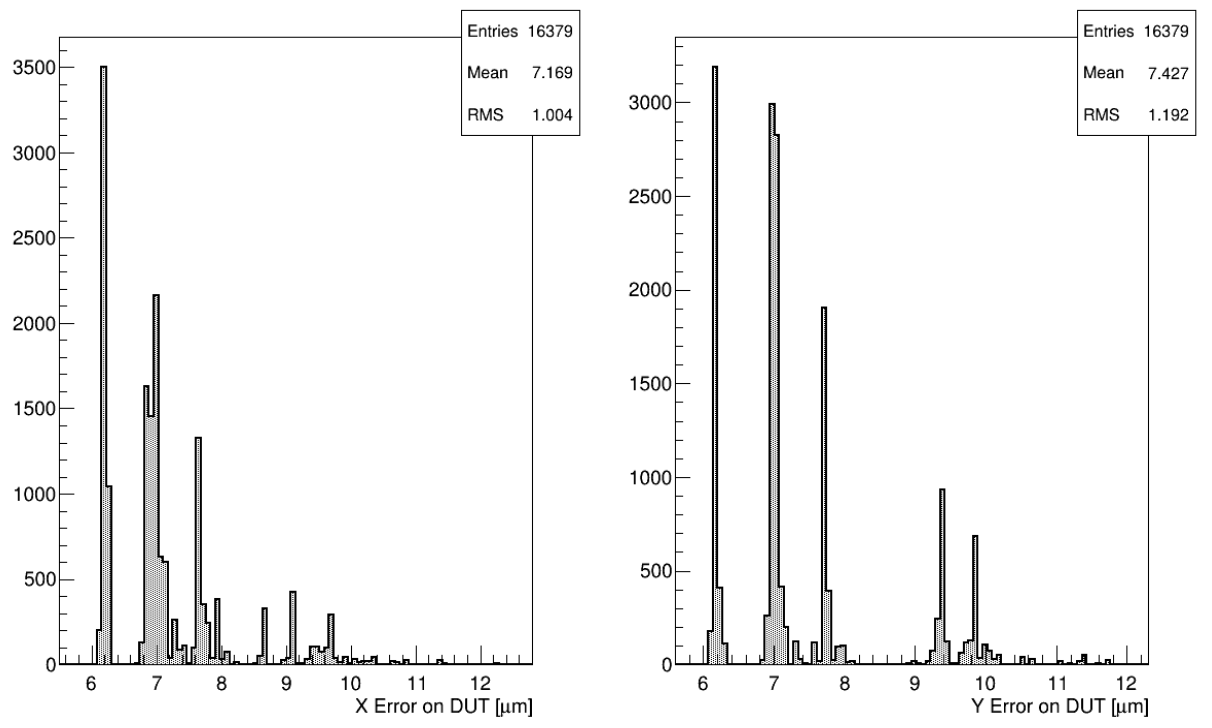

Figure 14. Track fit extrapolated error distributions at the DUT $Z$ position $(Z \approx 0)$ after a complete alignment. The discrete peaks result from the combinations of different hit resolution that can be associated to the tracks.

It turns out that the best achievable telescope resolutions on the DUT is as small as $6.2 \mu \mathrm{m}$ in both $\mathrm{X}$ and $\mathrm{Y}$ coordinates, and that the bulk of tracks give resolutions better 
than $8 \mu \mathrm{m}$. Excluding tracks with extrapolation error larger than $8 \mu \mathrm{m}$, as it is typically done for data analysis, the average error at DUT is approximately $6.9 \mu \mathrm{m}$ on both projections $(6.87 \mu \mathrm{m}$ in $\mathrm{X}$ and $6.94 \mu \mathrm{m}$ in $\mathrm{Y})$. This error will be called Pure Telescope extrapolation error.

To obtain the Total Expected extrapolation error (at $120 \mathrm{GeV}$ proton energy), one has to add in quadrature the error due to the Multiple Coulomb Scattering (MCS) in the DUT. For three or less DUTs placed in the standard configuration around the telescope center at $Z_{1}=+5 \mathrm{~cm}, Z_{2}=-5 \mathrm{~cm}$ and $Z_{3}=-6 \mathrm{~cm}$, the Total Expected extrapolation error is given by

$$
\sigma_{X}=\sqrt{6.87^{2}+1.37^{2} \times L_{D U T_{1}}+1.95^{2} \times L_{D U T_{2}}+2.01^{2} \times L_{D U T_{3}}} \mu \mathrm{m}
$$

\section{Performance in Beam Tests}

The test-beam performance of the telescope in testing a DUT and resolving its details was studied on several occasions during the numerous test-beam campaigns that were

$$
\sigma_{Y}=\sqrt{6.94^{2}+2.10^{2} \times L_{D U T_{1}}+2.68^{2} \times L_{D U T_{2}}+2.74^{2} \times L_{D U T_{3}}} \mu \mathrm{m}
$$

$L_{D U T_{n}}$ is the thickness of the nth DUT in percent of a radiation length. The errors in the previous formulae are negligible $(<0.1 \mu \mathrm{m})$ and hence omitted. conducted on different pixel sensors. Here, only the most critical figure is reported and, namely, the actual measured track extrapolation precision in real experimental conditions.

As a general criterion for the analysis of the beam test results, any event must simultaneously fulfill the following requirements: only one telescope track is reconstructed; the track must have hits on all the planes; no hits other than those associated to the track can be present on the telescope planes; and the Pure Telescope extrapolation error at the DUT position is less than $8 \mu \mathrm{m}$ in both views.

In Figure 15 the measured residual of a normally incident telescope track with respect to a doublet of adjacent hits on a silicon 3D pixel detector is reported. The coordinate attributed to the doublet was exactly the divide between the two adjacent pixels.

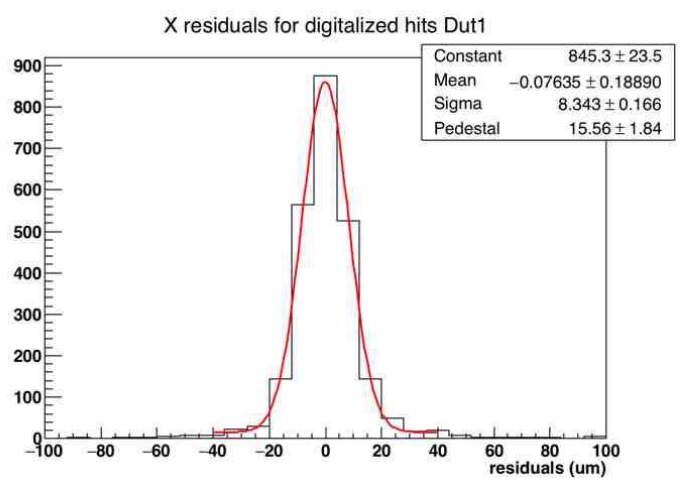

Figure 15. Track residuals of two adjacent hits along $X$ on the 3D Si-pixel detector. 
The silicon 3D pixel sensor (Sintef $2 \mathrm{E}$ ) had a $100 \times 150 \mu \mathrm{m}^{2}$ pixel cell with two central columnar signal-electrodes (10 $\mu \mathrm{m}$ radius) and six columnar field-electrodes ( $7 \mu \mathrm{m}$ radius) on the periphery. The sensor thickness was approximately $220 \mu \mathrm{m}$.

The measured RMS of the residual, $8.34 \pm 0.17 \mu \mathrm{m}$, results from the sum in quadrature of the actual track extrapolation error and that of the coordinate measured on the DUT. From a study of the correlation between the telescope track impact point and the charge asymmetry of the two pixels of the doublet on the DUT, it is found that the effective region of sharing is concentrated around the divide of the doublet with RMS $=2.38 \pm 0.60 \mu \mathrm{m}$. Unfolding this error from the measured residual, the actual track extrapolation error results $7.99 \pm 0.25 \mu \mathrm{m}$ and it is fully consistent with a Total Expected value of $7.86 \mu \mathrm{m}$, resulting from the sum in quadrature of the Pure Telescope resolution, $6.87 \mu \mathrm{m}$, and a multiple Coulomb scattering error of $3.82 \mu \mathrm{m}$, due to the simultaneous presence of three DUTs ( $\sim 1.5 \%$ of a radiation length each). On this basis one can conclude that the actual track extrapolation error at the DUT is well matched by the Total Expected extrapolation error resulting from the formulae in the previous section. For instance, the Total Expected extrapolation error for a typical test of a single DUT (1.5\% of a radiation length) is $7.07 \mu \mathrm{m}$ in X and $7.40 \mu \mathrm{m}$ in Y. Obviously, this performance could be further improved by selecting only the best resolution tracks and, specifically, those belonging to the first peak of Fig. 14 at 6.2 $\mu \mathrm{m}$.

Summarizing, the present telescope allows for a real track extrapolation error at the DUT typically better than $8 \mu \mathrm{m}$ both in $\mathrm{X}$ and $\mathrm{Y}$. This figure includes all experimental effects that can affect the precision during a long Run of 24 hours at least such as thermal excursion, vibrations and all the other possible phenomena.

\section{Conclusions}

This paper describes the pixel silicon telescope installed at the Fermilab Test Beam Facility (FTBF) that provides high resolution tracking of the beam particles. The impact point of the available $120 \mathrm{GeV}$ beam protons at the center of the telescope can be reconstructed with an accuracy of $8 \mu \mathrm{m}$, at least, on both the transversal coordinates. The detector hardware and control software is complemented by the Monicelli software, which provides to the users the reconstructed tracks necessary for their data analysis. The telescope has already been used by several experiments and, in particular, has been extensively used by the CMS forward pixel community to test sensor candidates for future CMS pixel upgrades [7-15].

\section{Acknowledgements}

We wish to thank the Fermilab Test Beam Facility personnel, and in particular Aria Soha, Mandy Rominsky and Eugene Schmidt, for the continuous support they provide us. This research was supported, in part, by the U.S. Department of Energy and the Italian Istituto Nazionale di Fisica Nucleare and Ministero della Ricerca Scientifica e Tecnologica.

We also wish to thank the LHC Physics Center (LPC) for the support given to our students Stefano Terzo, Jennifer Ngadiuba and Luigi Vigani. 
370 [1] Fermilab Test Beam Facility web site: http://www-ppd.fnal.gov/FTBF

[2] I.Rubinskiy: An EUDET/AIDA pixel beam telescope for detector development. Physics Procedia 37 ( 2012 ) 923 - 931

[3] I.Rubinskiy: A EUDET/AIDA pixel beam telescope as a tool for testing tracking detectors. Instrumentation Seminar at DESY (March 15, 2013)

[4] H. Chr. Kaestli et al.: Design and Performance of the CMS Pixel Detector Readout Chip. Nucl.Instrum.Meth. A565 (2006) 188-194

[5] Marcos Turqueti, Ryan A. Rivera, Alan Prosser, Jeffry Andresen, and John Chramowicz. CAPTAN: A hardware architecture for integrated data acquisition, control, and analysis for detector development. In Nuclear Science Symposium Conference Record, 2008. NSS '08. IEEE, pages 3546-3552.

[6] Ryan A. Rivera, Marcos Turqueti, and Alan Prosser. A software solution for the control, acquisition, and storage of CAPTAN network topologies. In Nuclear Science Symposium Conference Record, 2008. NSS '08. IEEE, pages 805-808.

[7] Mayur Bubna et al.: Laboratory and testbeam results for thin and epitaxial planar sensors for HL-LHC JINST 10.1088/1748-0221/10/08/C08002 C08002 1008 2015 2015-08-04.

[8] Mayur Bubna,et al.: Testbeam and Laboratory Characterization of CMS 3D Pixel Sensors JINST 10.1088/1748-0221/9/07/C07019 C07019 920142014

[9] Luigi Moroni et al.: Tracking performance of a single-crystal and a polycrystalline diamond pixel-detector JINST 10.1088/1748-0221/8/06/P06006 P06006 8 Solid state detectors Diamond Detectors Radiation-hard detectors Particle tracking detectors (Solid-state detectors) 2013 2013-06-01

[10] Alexander Krzywda et al.: Pre- and post-irradiation performance of FBK 3D silicon pixel detectors for CMS Nucl.Instrum.Meth. 10.1016/j.nima.2014.06.029 404-411 A763.

[11] Enver Alagoz et al.: Pre- and post-irradiation performance of FBK 3D silicon pixel detectors for CMS Nucl.Instrum.Meth. 10.1016/j.nima.2014.06.029 404-411 A763.

[12] Enver Alagoz et al. Testbeam and laboratory test results of irradiated 3D CMS pixel detectors Nucl.Instrum.Meth. 10.1016/j.nima.2013.07.042 52-56 A732.

[13] Ada Solano et al.: Performance of CMS 3D silicon pixel detectors before and after irradiation Nucl.Instrum.Meth. 10.1016/j.nima.2013.04.048 33-37 A730.

[14] Obertino Maria et al.: 3D-FBK pixel sensors with CMS readout: First test results Nucl.Instrum.Meth. 10.1016/j.nima.2012.11.076 342-344 A718.

[15] Lorenzo Uplegger et al.: Test-beam studies of diamond sensors for SLHC Nucl.Instrum.Meth. 10.1016/j.nima.2012.10.011 376-379 A718. 